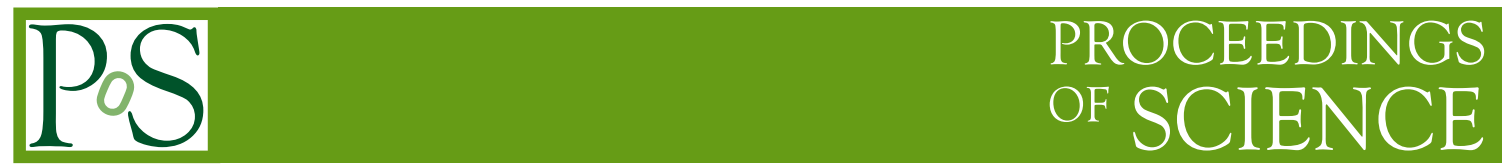

\title{
Search for New Physics with rare decays at CMS
}

\section{Ozlem Ozcelik* on behalf of CMS Collaboration}

Bogazici University

E-mail: ozlem.ozcelik@cern.ch

One of the major goals of the LHC experiments is probing for new physics beyond Standard Model. We report the most recent experimental results to search for new physics in rare $B$ mesons decaying to dimuons and searches of the $\tau$ leptons decaying to three muons in $W$ boson and heavy-flavor boson channels at CMS.

40th International Conference on High Energy physics - ICHEP2020

July 28 - August 6, 2020

Prague, Czech Republic (virtual meeting)

\footnotetext{
${ }^{*}$ Speaker
} 


\section{Search for rare $B \rightarrow \mu^{+} \mu^{-}$decays}

The Standard Model (SM) expectations for the branching fractions of $B_{s}^{0} \rightarrow \mu^{+} \mu^{-}$and $B^{0} \rightarrow$ $\mu^{+} \mu^{-}$rare decays are very suppressed since they are flavor-changing-neutral-current (FCNC) decays that can only occur via $\mathrm{Z}$ penguin and box diagrams. The branching fractions are expected to be $\mathcal{B}\left(B_{s}^{0} \rightarrow \mu^{+} \mu^{-}\right)=(3.66 \pm 0.14) \times 10^{-9}$ and $\mathcal{B}\left(B^{0} \rightarrow \mu^{+} \mu^{-}\right)=(1.03 \pm 0.05) \times 10^{-10}$ in SM [1, 2]. However, any effect beyond SM can enchance these branching fractions significantly [3, 4]. Another observable which is also sensitive to new physics effects is $B_{s}^{0} \rightarrow \mu^{+} \mu^{-}$effective lifetime. SM predicts that only heavy mass eigenstate of $B_{s}^{0}$, with a lifetime of $\tau_{B_{s H}^{0}}=1.615 \pm 0.009 \mathrm{ps}$ [5], can decay to dimuon. It is therefore of high interest to measure, in addition to the branching fraction, also the $B^{0} \rightarrow \mu^{+} \mu^{-}$effective lifetime.

The signal events of $B \rightarrow \mu^{+} \mu^{-}$are reconstructed using two oppositely charged muons fitted to a common displaced vertex, with an invariant mass $m_{\mu \mu}$ around the $B^{0}$ or $B_{s}^{0}$ mass. The main sources of the background is the combinatorial background as including either two uncorrelated semileptonic $B$ decays or one semileptonic $B$ decay plus one misidentified hadron. Another background component is the rare single $B$ decays which consist of decays with either one (e.g., $B \rightarrow h \mu v)$ or two misidentified hadron $(B \rightarrow h h$ ) leaking into signal region. Due to the (very) small expected signal rate, it is crucial to suppress the large background which their rates are even higher than the signal events. The best possible hadron-to-muon misidentification probability is achieved using an advanced muon identification study, based on a Boosted Decision Tree (BDT), using the variables from tracking and the muon detectors.

A second BDT is trained for the candidate selection using signal decays from Monte Carlo (MC) simulations and combinatorial background from data sidebands. The BDT categorization is optimized by maximizing the expected sensitivity of the analysis as choosing the best significance for the branching fraction and the smallest uncertainty for the lifetime analysis using a maximum likelihood estimator.

The normalization yield is obtained from a fit to $J / \psi K$ invariant mass distribution. The signal shape is modelled with double Gaussians with a common mean. The combinatorial background component is an exponential function, an error function for partially reconstructed $B \rightarrow J / \psi K^{+} X$ decays and a MC simulation based shape for the $B^{+} \rightarrow J / \psi \pi^{+}$is fixed to $4 \%$ of the total signal yield.

The branching fraction is measured relative to a normalization channel $B^{+} \rightarrow J / \psi K^{+}$, as $\mathcal{B}\left(B_{s}^{0} \rightarrow \mu^{+} \mu^{-}\right)=\frac{N_{\text {sig }}}{N\left(B^{+} \rightarrow J / \psi K^{+}\right)} \times \mathcal{B}\left(B_{s}^{0} \rightarrow J / \psi\left(\rightarrow \mu^{+} \mu^{-}\right) K^{+}\right) \times \frac{\varepsilon^{t o t}\left(B^{+}\right)}{\varepsilon^{\text {tot }}\left(B_{s}^{0}\right)} \times \frac{f_{u}}{f_{s}}$, where $N_{\text {sig }}$ $\left(N\left(B^{+}\right)\right)$is the signal (normalization) decays, $\varepsilon^{t o t}\left(B_{s}^{0}\right)\left(\varepsilon^{t o t}\left(B^{+}\right)\right)$is the signal $\left(B^{+}\right)$efficiency, $\mathcal{B}\left(B_{s}^{0} \rightarrow J / \psi K^{+}\right)=(1.01 \pm 0.03) \times 10^{-3}$ and $\mathcal{B}\left(J / \psi \rightarrow \mu^{+} \mu^{-}\right)=(5.96 \pm 0.03) \times 10^{-2}$ and $f_{u} / f_{s}$ is the fragmentation fractions of the $\left(B^{+}\right)$and $B_{s}^{0}$ mesons. A similar formula is used for $\mathcal{B}\left(B^{0} \rightarrow \mu^{+} \mu^{-}\right)$, using $f_{d} / f_{u}=1$.

The branching fractions $\mathcal{B}\left(B_{s}^{0} \rightarrow \mu^{+} \mu^{-}\right)$and $\mathcal{B}\left(B^{0} \rightarrow \mu^{+} \mu^{-}\right)$are determined with a 3D unbinned maximum likelihood fit to the dimuon invariant mass, the relative mass resolution, and the dimuon pairing configuration $C$ ( $C= \pm 1$ for the two muons bending towards or away from each other respectively.) The signal probability density functions (PDFs) are modelled with a Crystal Ball function for the invariant mass where the Gaussian width is scaled according to $\sigma\left(m_{\mu \mu}\right)$. The peaking background PDF is described by a sum of Crystal-ball function and a Gaussian (a 
non-parametric keys model with Gaussian kernel). The combinatorial background is modelled by a nonnegative Bernstein polynomial of the first degree, whose parameters (both slope and normalization) are determined in the fit. The result of the fit to data is

$$
\mathcal{B}\left(B_{s}^{0} \rightarrow \mu^{+} \mu^{-}\right)=\left[2.9 \pm 0.7(\exp ) \pm 0.2\left(\mathrm{f}_{\mathrm{s}} / \mathrm{f}_{\mathrm{u}}\right)\right] \times 10^{-9}
$$

where the experimental uncertainty is a combination of large statistical and a small systematic errors and the second one is due to the uncertainty in $f_{s} / f_{u}$. The observed (expected) significance, determined using Wilks' theorem [6], is 5.6 (6.5) standard deviations. Since no significant signal is observed, an upper limit is set for the $\mathcal{B}\left(B^{0} \rightarrow \mu^{+} \mu^{-}\right)$based on the standard $\mathrm{CL}_{\mathrm{s}}$ rule [7]. The result is $\mathcal{B}\left(B^{0} \rightarrow \mu^{+} \mu^{-}\right)<3.6 \times 10^{-10}\left(3.1 \times 10^{-10}\right)$ at the $95 \%(90 \%)$ confidence level. The invariant mass distributions with the fit projection overlays, as well as the likelihood contours, are shown in Fig 1.

The effective lifetime is measured with two methods and as a primary method, 2D unbinned maximum likelihood fit is performed to the invariant mass distribution and the proper decay time in the range of $1<t<11$ ps. The combined distributions from all categories are shown in Fig. 2 and the fit gives the result as

$$
\tau_{\mu \mu}=1.70_{-0.44}^{+0.61} \mathrm{ps}
$$

where the uncertainty is the combination of large statistical and small systematic errors $( \pm 0.09 \mathrm{ps})$.

This is the first time CMS performed effective lifetime analysis for $B_{s}^{0} \rightarrow \mu^{+} \mu^{-}$decays. All results are found to be consistent with SM predictions.
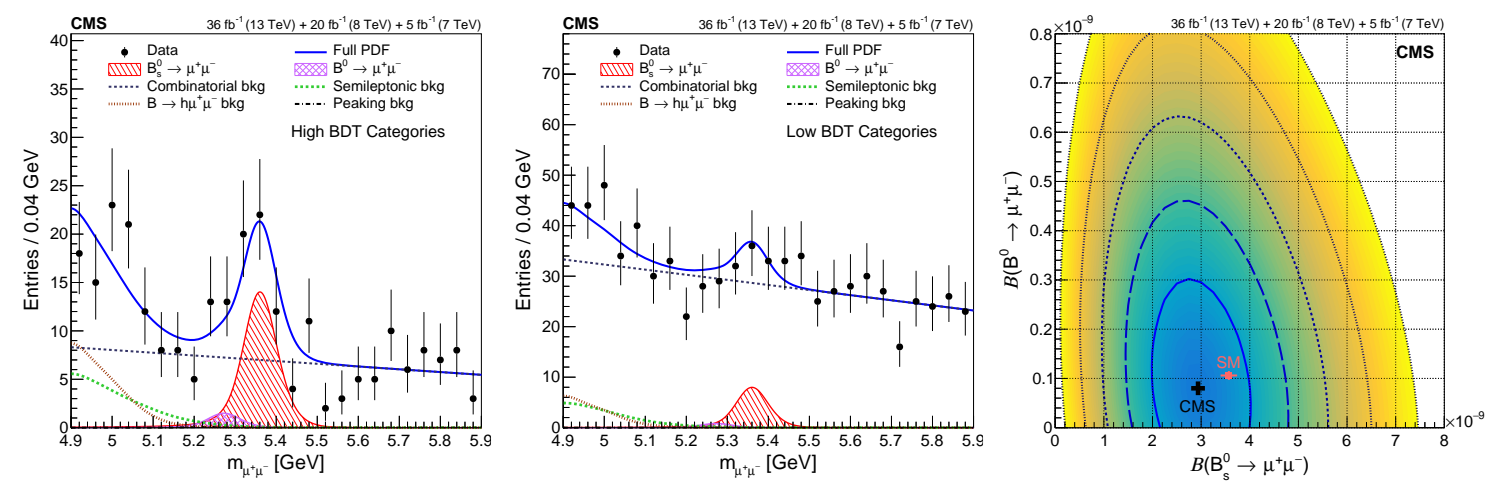

Figure 1: Combined mass projections for the high (left) and low (middle) BDT-threshold categories. Right plot shows the likelihood contours for the fit to the branching fractions together with the best-fit value (cross) and the SM expectation (solid square). Details can be found in the original paper [8].

\section{Search for $\tau \rightarrow 3 \mu$ decays}

The search exploits $\tau$ leptons in both $W$ boson and heavy-flavor hadron decays. It's been observed that neutrinos have masses and they can oscillate through generations which provides a 

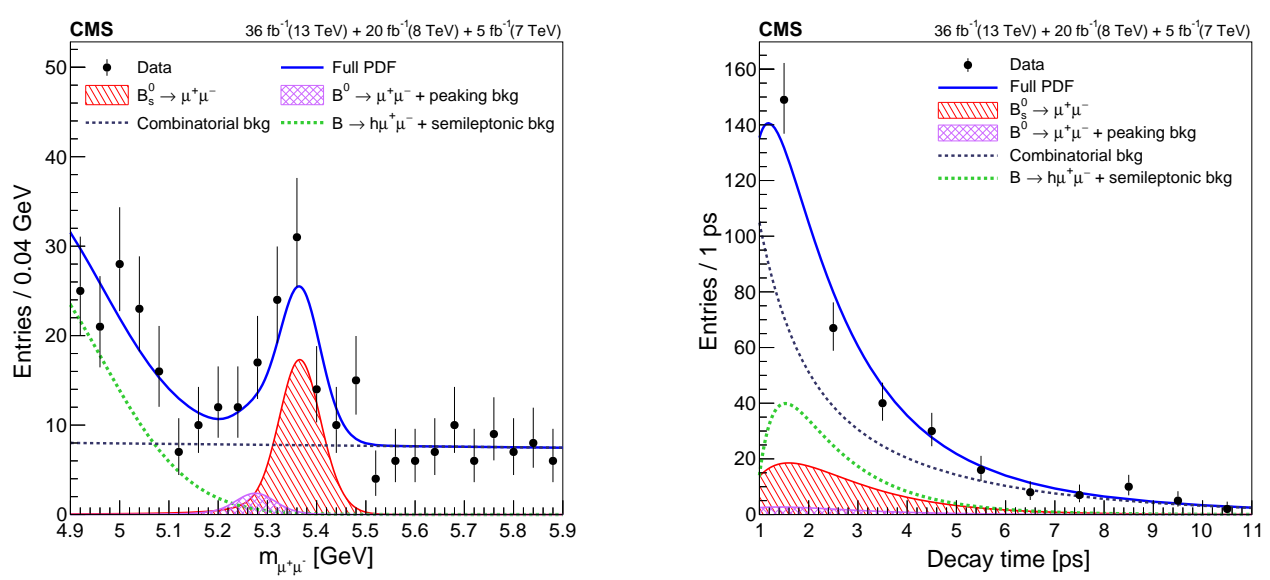

Figure 2: Invariant mass (left) and proper decay time (right) distributions, with the 2D UML fit projections overlaid. The total fit is shown by the solid line and the different back- ground components by the broken lines and cross-hatched distributions. The signal component is shown by the single-hatched distribution. Details can be found in the original paper [8].

mechanism for lepton flavor violating (LFV) decays of $\tau \rightarrow 3 \mu$. However, the SM expectation for the branching fraction is extremely small [9]. This rate can be altered by new physics scenarios [10, 11]. CMS provides the first search for the LFV decay from a combination of two independent channels.

\section{1 $W$ boson channel}

$\tau \rightarrow 3 \mu$ is obtained from $W \rightarrow \mu \nu$ decays, where $v$ is the tau-neutrino that appears as a missing transverse energy, $E_{\text {miss }}^{T}$, in the decay. The high-level trigger required three reconstructed charged particles (tracks), of which two must be identified as muons with $p_{T}>3 \mathrm{GeV}$ and the other must have $p_{T}>1.2 \mathrm{GeV}$. Offline, all combinations of three muons in the event with a combined charge of \pm 1 are considered and a fit to a common vertex is attempted to make a $\tau$ candidate.

The branching fraction of the $\tau \rightarrow 3 \mu$ is obtained as $\mathcal{B}(\tau \rightarrow 3 \mu)=\frac{N_{\mathrm{sig}}}{\mathcal{L} \sigma(p p \rightarrow W+X) \mathcal{B}(W \rightarrow \tau \nu) \mathrm{A}_{3 \mu} \varepsilon_{3 \mu} \mu}$, where number of signal events are normalized using integrated luminosity, $W$ cross section, the branching fraction of $W \rightarrow \tau v$ and the corresponding acceptance and efficiencies, respectively.

The events passing the BDT selection are classified into two mutually exclusive categories, based on trimuon mass resolution in barrel $\left(\left|\eta_{\tau}\right|<1.6\right)$ and endcap $\left(\left|\eta_{\tau}\right| \geq 1\right.$.6) regions of the detector. The trimuon invariant mass distributions for each events passing each category is shown in Fig. 3, along with a background-only fit and the contribution expected for a signal with $\mathcal{B}(\tau \rightarrow 3 \mu)=10^{-7}$. For the $W$ boson analysis, the signal model is a Gaussian function with fixed mean and width, as determined from fitting the simulated events in the appropriate category.

\subsection{Heavy-flavor hadron channel}

The search for $\tau \rightarrow 3 \mu$ produced in $B$ and $D$ meson decays requires a normalization channel for the measurement of the branching fraction in order to reduce large systematic uncertainties in the production of heavy-flavor hadrons. For this purpose, the decay $D_{s}^{+} \rightarrow \phi \pi^{+} \rightarrow \mu^{+} \mu^{-} \pi^{+}$is used to normalize the signal yield. A $\tau$ candidate is obtained from two reconstructed muons plus one track at the trigger level. Then, it applies trimuon invariant mass requirement and the sum of 



Figure 3: Trimuon invariant mass distributions for barrel (left) and endcap (right) categories of the $W$ boson analysis. The background-only fit and the expected signal yield assuming $\mathcal{B}(\tau \rightarrow 3 \mu)=10^{-7}$ are shown with solid and dashed lines, respectively. Details can be found in the original paper [12].

charges of these trimuon to be \pm 1 at the offline selection. Since $D_{s}$ hadron can also be produced from a $B$ decay, the fraction of non-prompt $D_{s}$ events is calculated from a fit to proper decay length distribution.

To improve the analysis sensitivity, the $\tau$ candidated are divided into six categories depending on their trimuon mass resolution and the BDT score. A simultaneous maximum likelihood fit to the trimuon invariant mass, is performed with all six categories, as shown in Fig 4. The signal model is parametrized with Crystal-Ball functions, while the background component is modeled with an exponential plus a polynomial.

\subsection{Results}

CMS offers the first search of LFV decay $\tau \rightarrow 3 \mu$ from a combination of $W$ and heavy-flavor channel. No signal is observed and upper limit is extracted from simultaneous unbinned maximum likelihood fit to the trimuon invariant mass in the two categories of the $W$ boson analysis and the six categories of the heavy-flavor analysis. The observed (expected) upper limits at $90 \% \mathrm{CL}$ on $\mathcal{B}(\tau \rightarrow 3 \mu)$ is $8.0 \times 10^{-8}\left(6.9 \times 10^{-8}\right)$.

\section{References}

[1] C. Bobeth, M. Gorbahn, T. Hermann, M. Misiak, E. Stamou and M. Steinhauser. Phys. Rev. Lett. 112, 101801 (2014) doi:10.1103/PhysRevLett.112.101801 [arXiv:1311.0903 [hep-ph]].

[2] M. Beneke, C. Bobeth and R. Szafron, Phys. Rev. Lett. 120, no.1, 011801 (2018) doi:10.1103/PhysRevLett.120.011801 [arXiv:1708.09152 [hep-ph]].

[3] K. S. Babu and C. F. Kolda. Phys. Rev. Lett. 84 (2000) 228, arXiv:hep-ph/9909476.

[4] G. Isidori and A. Retico. JHEP 11 (2001) 001, arXiv:hep-ph/0110121. 

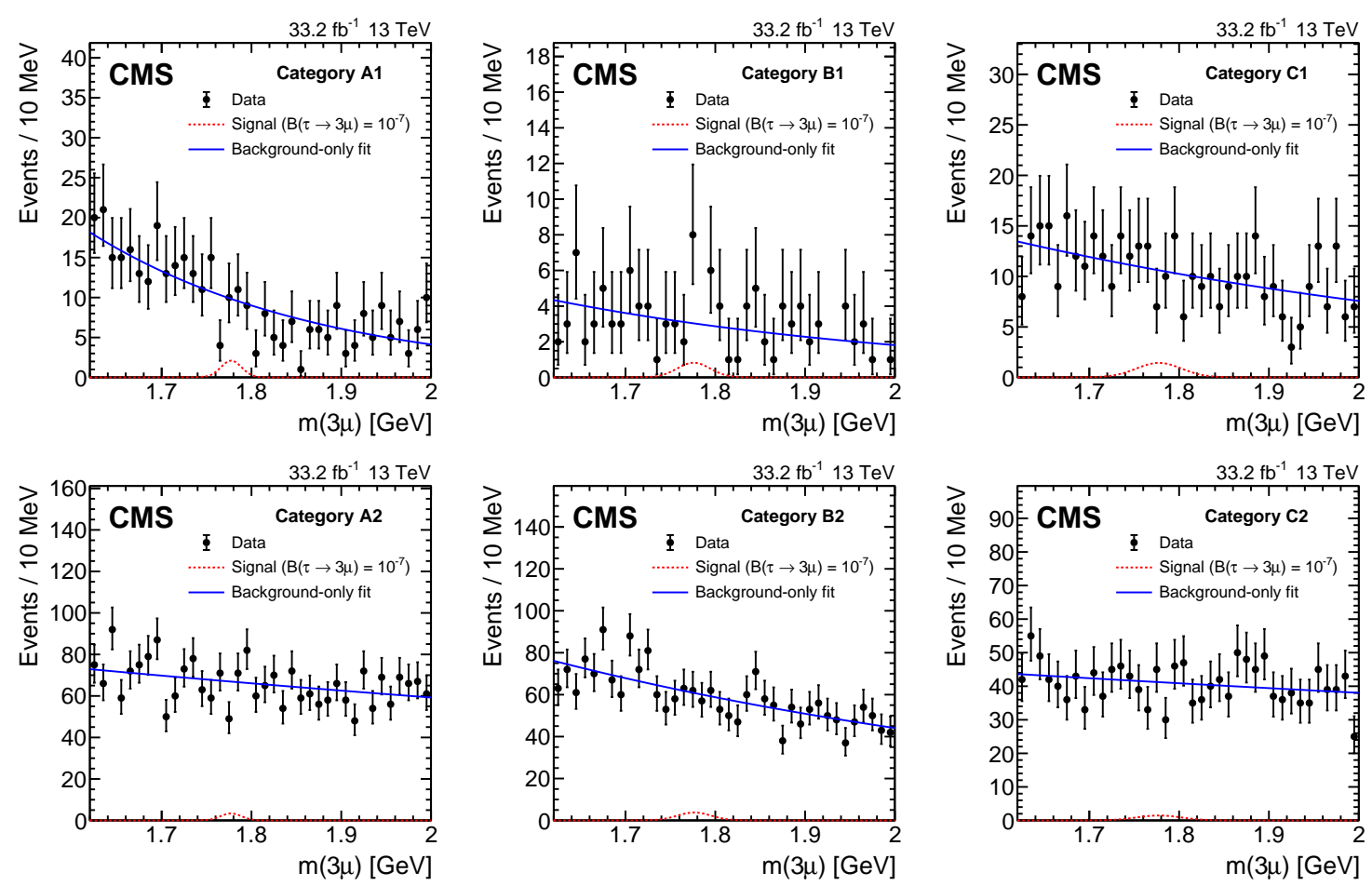

Figure 4: Trimuon mass distributions in the six independent event categories used in the heavy-flavor channel. The background-only fit and the expected signal yield assuming $\mathcal{B}(\tau \rightarrow 3 \mu)=10^{-7}$ are shown with solid and dashed lines, respectively. Details can be found in the original paper [12].

[5] Particle Data Group, M. Tanabashi et al. Phys. Rev. D 98 (2018) 030001, doi:10.1103/PhysRevD.98.030001

[6] S. S. Wilks. Annals Math. Statist. 9 (1938) 60, doi:10.1214/aoms/1177732360.

[7] A. L. Read. J. Phys. G 28 (2002) 2693, doi:10.1088/0954-3899/28/10/313.

[8] CMS Collaboration. JHEP 04 (2020) 188, doi:10.1007/JHEP04(2020)188, arXiv:1910.12127.

[9] X.-Y. Pham. Eur. Phys. J. C 8 (1999) 513, doi:10.1007/s100529901088, arXiv:hep$\mathrm{ph} / 9810484$.

[10] W. J. Marciano, T. Mori, and J. M. Roney. Ann. Rev. Nucl. Part. Sci. 58 (2008) 315, doi:10.1146/annurev.nucl.58.110707.171126.

[11] E. Arganda and M. J. Herrero. Phys. Rev. D 73 (2006) 055003, doi:10.1103/PhysRevD.73.055003, arXiv:hep-ph/0510405.

[12] CMS Collaboration. CMS-BPH-17-004. arXiv:2007.05658 [hep-ex]. 